BULL. AUSTRAL. MATH, SOC.

VOL. 34 (1986) 211-218

\title{
ON AN INTEGRAL OPERATOR FOR CONVEX UNIVALENT FUNCTIONS
}

\section{Vinod KUMAR AND S.L. SHUKLA}

Let $K(m, M)$ denote the class of functions $f(z)=z+\sum_{n=2}^{\infty} a_{n} z^{n}$ regular and satisfying $\left|1+z f^{\prime \prime}(z) / f^{\prime}(z)-m\right|<M$ in $|z|<1$, where $|m-1|<M \leq m$. Recently, R.K. Pandey and G. P. Bhargava have shown that if $f \in K(m, M)$, then the function $F(z)=\int_{0}^{z}\left\{f^{\prime}(u)\right\}^{\alpha} d u$ also belongs to $K(m, M)$ provided $\alpha$ is a complex number satisfying the inequality $|\alpha| \leq(1-b) / 2$, where $b=(m-1) / M$. In this paper we show by a counterexample that their inequality is in general wrong, and prove a corrected version of their result. We show that $F \in K(m, M)$ provided that $\alpha$ is a real number satisfying $-\phi \leq \alpha \leq 1, \phi=(M-|m-1|) /$ $(M+|m-1|)$, or a complex number satisfying $|\alpha| \leq \phi$. In both cases the bounds for $\alpha$ are sharp.

1. Introduction

Let $S$ denote the class of functions $f(z)=z+\sum_{n=2}^{\infty} a_{n} z^{n}$ which

Received 12 November 1985.

Copyright Clearance Centre, Inc. Serial-fee code: 0004-9727/86 $\$ A 2.00+0.00$. 
are regular in the unit disc $U=\{z:|z|<1\}$. A function $f$ of $S$ belongs to the class $S(m, M)$ if $\left|z f^{\prime}(z) / f(z)-m\right|<M$ for $z \in U$, where $|m-1|<M \leq m$. A function $f$ of $S$ belongs to the class $K(m, M)$ if $\left|1+z f^{\prime \prime}(z) / f^{\prime}(z)-m\right|<M$ for $z \in U$. Evidently, the functions in $S(m, M)$ and $K(m, M)$ are starlike and convex univalent respectively. These classes were introduced by Jakubowski [1],[2].

Recently, Kumar and Shukla [4] have studied class preserving integral operators for $S(m, M)$, and called them 'Jakubowski starlike integral operators'. It is now natural to consider a similar problem for the class $K(m, M)$. We define:

An integral operator which maps the class $K(m, M)$ into or onto itself is called a Jakubowski convex integral operator.

The object of this paper is to show that, for suitable choices of constant $\alpha$, the integral operator

$$
I(f)=\int_{0}^{z}\left\{f^{\prime}(u)\right\}^{\alpha} d u
$$

maps $K(m, M)$ into itself. One of our results improves and corrects a recent result of Pandey and Bhargava [5].

\section{Fundamental Lemmas.}

In this section we prove two lemmas which play an important role in establishing a theorem concerning the Jakubowski convex integral operators. The first one is equivalent to Lemma 2.3 in [4] when. $\beta=1$. However we present the details of the proof since we require it in the discussion which follows.

LEMMA 2.1. Let $\alpha, m$ and $M$ be real numbers such that $0<\alpha \leq 1$ and $|m-1|<M \leq m$. If

$$
t=1-\alpha+\alpha m \text { and } T=\alpha M,
$$

then $K(t, T) \subseteq K(m, M)$.

Proof. It suffices to show that

$$
m-M \leq t-T \text { and } t+T \leq m+M \text {. }
$$

We need consider only the case when $0<a<1$. Suppose $m-M>t-T$. Then, $m-M>1-\alpha+\alpha(m-M)$, which implies that $m-M>1$. But this is contrary to the assumption $|m-1|<M$. Next, suppose $t+T>m+M$. 
Then, $1-\alpha+\alpha(m+M)>m+M$, which implies $m+M<1$. This is also contrary to $|m-1|<M$. Therefore the inequalities (2.2) hold.

LEMMA 2.2 Let $a, m$ and $M$ be real numbers such that

$$
\max \left\{-\frac{1-(m-M)}{m+M-1},-\frac{m+M-1}{1-(m-M)}\right\} \leq \alpha<0
$$

and $|m-1|<M \leq m$. If

$$
\tilde{t}=1-\alpha+\alpha m \text { and } \widetilde{T}=-\alpha M \text {, }
$$

then $K(\tilde{T}, \tilde{T}) \subseteq K(m, M)$.

Proof. We need to show that

$$
m-M \leq \tilde{t}-\tilde{T} \text { and } \tilde{t+\tilde{T}} \leq m+M \text {. }
$$

suppose $m-M>\tilde{t}-\tilde{T}$. Then, $m-M>1-\alpha+\alpha(m+M)$, which implies that $\alpha<-(1-(m-M)) /(m+M-1)$. Further, suppose $\tilde{t}+\tilde{T}>m+M$. Then, $1-\alpha+\alpha(m-M)>m+M$, which implies $\alpha<-(m+M-1) /(1-(m-M))$.

Now if

$$
\alpha<\max \left\{-\frac{1-(m-M)}{m+M-1},-\frac{m+M-1}{1-(m-M)}\right\},
$$

then at least one of the inequalities in (2.5) does not hold. Whence the disc centred at $\tilde{t}$ and having radius $\tilde{T}$ is not contained in the disc centred at $m$ and having radius $M$. Therefore $K(\tilde{t}, \tilde{T}) \subseteq K(m, M)$ if $\alpha$ satisfies $(2.3)$.

It is noticeable that the truth of $|\tilde{t}-1|<\tilde{T} \leq \tilde{t}$ requires $\alpha \geq-1 / m+M-1)$, which automatically holds since

$$
\begin{aligned}
\alpha & \geq \max \left\{-\frac{1-(m-M)}{m+M-1},-\frac{m+M-1}{1-(m-M)}\right\} \\
& \geq-(1-(m-M)) /(m+M-1) \\
& \geq-1 /(m+M-1) .
\end{aligned}
$$

NOTE. From now on $t, T$ and $\tilde{t}, \tilde{T}$ will be as in (2.1) and (2.4) respectively.

\section{Jakubowski Convex Integral Operators.}

In this section we obtain the main results of this paper. 
THEOREM 3.1. If $f \in K(m, M)$, then the function $F$, defined by

$$
F(z)=\int_{0}^{z}\left\{f^{\prime}(u)\right\}^{\alpha} d u,
$$

also belongs to $K(m, M)$, provided

where $\phi=(M-|m-1|) / M+|m-1|)$.

The result is sharp.

Proof. From (3.1) we have

$$
F^{\prime}(z)=\left\{f^{\prime}(z)\right\}^{\alpha} .
$$

Logarithmic differentiation yields

$$
1+z \frac{F^{\prime \prime}(z)}{F^{\prime}(z)}=\alpha\left\{1+z \frac{f^{\prime \prime}(z)}{f^{\prime}(z)}\right\}+(1-\alpha) .
$$

Therefore

$$
1+z \frac{F^{\prime \prime}(z)}{F^{\prime}(z)}-(1-\alpha+\alpha m)=\alpha\left[1+z \frac{f^{\prime \prime}(z)}{f^{\prime}(z)}-m\right\} .
$$

Now consider two cases, namely $\alpha>0$ and $\alpha<0$.

Case I. When $\alpha>0$, it follows from (3.3) that

$$
\begin{aligned}
\left|1+z \frac{F^{\prime \prime}(z)}{F^{\prime}(z)}-(1-\alpha+\alpha m)\right| & =\alpha\left|1+z \frac{f^{\prime \prime}(z)}{f^{\prime}(z)}-m\right| \\
& <\alpha M \text {, since } f \in K(m, M) .
\end{aligned}
$$

Thus $F \in K(t, T)$, and hence, by Lemma 2.1, $F \in K(m, M)$ provided

$$
0<\alpha \leq 1 \text {. }
$$

Case II. When $a<0$, from (3.3) we get

$$
\begin{aligned}
\left|1+z \frac{F^{\prime \prime}(z)}{F^{\prime}(z)}-(1-\alpha+\alpha m)\right| & =-\alpha\left|1+z \frac{f^{\prime \prime}(z)}{f^{\prime}(z)}-m\right| \\
& <-\alpha M .
\end{aligned}
$$

Thus $F \in K(\tilde{t}, \tilde{T})$, and hence, by Lemma $2.2, F \in K(m, M)$ provided

$$
\max \left\{-\frac{1-(m-M)}{m+M-1},-\frac{m+M-1}{1-(m-M)}\right\} \leq \alpha<0 .
$$

It is now easy to compute that

$$
\max \left\{-\frac{1-(m-M)}{m+M-1},-\frac{m+M-1}{1-(m-M)}\right\}= \begin{cases}-\frac{1-(m-M)}{m+M-1}, & \text { when } m>1 \\ -1, & \text { when } m=1 \\ -\frac{m+M-1}{1-(M-M)}, & \text { when } m<1 .\end{cases}
$$

The expressions on the right hand side are easily seen to be equal to - $\phi$. 
Hence, combining (3.4), (3.5) and the trivial case $\alpha=0$, we conclude that $F \in K(m, M)$ if $\alpha$ satisfies (3.2).

To establish the sharpness of the result we take

$$
f(z)= \begin{cases}f^{z}(1-b u)^{-(a+b) / b} d u, & \text { when } m \neq 1, \\ f^{z} e^{M u} d u, & , \text { when } m=1,\end{cases}
$$

where

$$
a=\frac{M^{2}-m^{2}+m}{M} \text { and } b=\frac{m-1}{M}
$$

It is easy to see that $1+z f^{\prime \prime}(z) / f^{\prime}(z)$ maps $U$ onto the disc centred at $m$ and having radius $M$. It follows then from (3.3) that $I+z F^{\prime \prime}(z) / F^{\prime}(z)$ maps $U$ onto the disc centred at $t$ and having radius $T$, when $\alpha>0$. Now if $\alpha>1$, none of the inequalities in (2.2) holds, and consequently the disc centred at $t$ and having radius $T$ is not contained in the disc centred at $m$ and having radius $M$. Therefore $F \notin K(m, M)$ if $\alpha>1$. Similarly we can show that $F \notin K(m, M)$ if $\alpha<-\phi$. Hence the result is sharp.

In the particular case when $m=M$ and $m \rightarrow \infty, K(m, M)$ is equal to the well known class $K$ of convex functions. Hence the following result of Kim and Merkes [3] follows from Theorem 3.1.

COROLLARY 3.1. If $f \in K$, then the function $F$ defined by (3.1) also belongs to $K$, provided $0 \leq \alpha \leq 1$. The result is sharp with the extremal function $f(z)=\int_{0}^{z}(1-u)^{-2} d u$.

Very recently, Pandey and Bhargava [5] have shown that if $f \in K(m, M)$, then the function $F$ defined by (3.1) also belongs to $K(m, M)$ provided $\alpha$ is a complex number such that $|\alpha| \leq(1-b) / 2$, where $b$ is given by (3.6). We show below that their result is incorrect:

Let us consider the function

$$
f(z)=\int_{0}^{z}(1-b u)^{-(a+b) / b} d u
$$

with $m=.7$ and $M=.6$, so that $a=.95$ and $b=-.5$. Evidently, $f \in K(.7, .6)$ and $(1-b) / 2=.75$. Now, by the abovementioned result of Pandey and Bhargava, $F \in K(.7, .6)$ provided $|\alpha| \leq .75$. But, if we take $\alpha=-.75$, then from (3.1) and (3.7) we are led to 


$$
\left|1+z \frac{F^{\prime \prime}(z)}{F^{\prime}(z)}-.7\right|=\left|-.75\left\{\frac{1+.95 z}{1+.5 z}\right\}+1.05\right| ;
$$

and at $z=-.8$, the right hand side of this equation is equal to .75 . Thus we see that

$$
\left|1+z \frac{F^{\prime \prime}(z)}{E^{\prime}(z)}-.7\right|>.6
$$

at some point in the unit disc $U$. Therefore $F \notin K(.7,6)$. Hence the result of Pandey and Bhargava [5] is incorrect.

We now proceed to improve and correct the result of Pandey and Bhargava [5]. First we prove:

THEOREM 3.2. If $f \in K(m, M)$, then the function $F$ defined by (3.1) also belongs to $K(m, M)$ provided a is a complex number satisfying

$$
|\alpha|<1, \frac{|1-\alpha|}{1-|\alpha|} \leq \frac{M}{|m-1|}=\frac{1+\phi}{1-\phi} \text {, when } m \neq 1
$$

cond

$$
\text { (3.9) }|\alpha| \leq 1 \text {, when } m=1 \text {, }
$$

where $\phi=(M-|m-1|) /(M+|m-1|)$.

Proof. We have (as in Theorem 3.1)

$$
1+z \frac{F^{\prime \prime}(z)}{F^{\prime}(z)}=\alpha\left\{1+z \frac{f^{\prime \prime}(z)}{f^{\prime}(z)}-m\right\}+(1-\alpha) .
$$

Therefore, when $m \neq 1$, we obtain

$$
\begin{aligned}
\left|1+z \frac{F^{\prime \prime}(z)}{F^{\prime}(z)}-m\right| & =\left|\alpha\left\{1+z \frac{f^{\prime \prime}(z)}{f^{\prime}(z)}-m\right\}-(1-\alpha)(m-1)\right| \\
& \leq|\alpha|\left|1+z \frac{f^{\prime \prime}(z)}{f^{\prime}(z)}-m\right|+|1-\alpha||m-1| \\
& <|\alpha| M+|1-\alpha||m-1| \\
& \leq M,
\end{aligned}
$$

provided

$$
|\alpha|<1 \text { and } \frac{|1-\alpha|}{1-|\alpha|} \leqslant \frac{M}{|m-1|}=\frac{1+\phi}{1-\phi}
$$

Further, when $m=1$, we obtain 


$$
\begin{aligned}
\left|1+z \frac{f^{\prime \prime}(z)}{f^{\prime}(z)}-1\right| & =\left|\alpha\left\{1+z \frac{f^{\prime \prime}(z)}{f^{\prime}(z)}-1\right\}\right|, \\
& =|\alpha|\left|1+z \frac{f^{\prime \prime}(z)}{f^{\prime}(z)}-1\right|, \\
& <|\alpha| M, \\
& \leq M,
\end{aligned}
$$

provided $|\alpha| \leq 1$. This completes the proof of the theorem.

Using the above theorem we now establish the following corollary which improves and corrects the result of Pandey and Bhargava [5]. It is worth noting that the technique employed by us is different; and the result is sharp also.

COROLLARY 3.2. If $f \in K(m, M)$, then the function $F$ defined by (3.1) also belongs to $K(m, M)$ provided $\alpha$ is a complex number satisfying (3.10)

$$
|\alpha| \leq \phi \text {. }
$$

The result is sharp in the sense that the region given by (3.10) cannot be extended into any larger disc centred at the origin on the $\alpha-p l a n e$.

Proof. When $m=1$, the inequality (3.10) is identical with the inequality (3.9). We need therefore consider only the case when $m \neq 1$. In this case, from (3.8), we have

$$
\frac{|1-\alpha|}{1-|\alpha|} \leq \frac{1+\phi}{1-\phi}
$$

This inequality is implied by

$$
\frac{1+|\alpha|}{1-|\alpha|} \leq \frac{1+\phi}{1-\phi}
$$

which is equivalent to

$$
|\alpha| \leq \phi
$$

Hence, by Theorem $3.2, F \in K(m, M)$ if $|\alpha| \leq \phi$.

To show the sharpness of the inequality (3.10), let us assume that $F \in K(m, M)$ for all values of $\alpha$ lying in a larger disc $|\alpha| \leq \phi+s$, for some $s>0$. Then, in particular, $F \in K(m, M)$ for all values of $\alpha$ lying in the annulus $\phi<|\alpha| \leq \phi+s$. From this it follows that when $\alpha$ is real and negative, then $F \in K(m, M)$ even if $-(\phi+s) \leq \alpha<-\phi$. But 
this is contrary to the sharpness of $\alpha \geq-\phi$ in (3.2). Therefore the region given by $(3,10)$ cannot be extended into any larger disc centred at the origin on the $\alpha$-plane. Hence the result is sharp.

Remark. We could not establish the sharpness of Theorem 3.2 when $m \neq 1$. However the result is sharp for $m=1$ (as shown in corollary 3.2). Therefore, it would be interesting to establish the sharpness of the inequality $(3.8)$.

\title{
References
}

[1] Zbigniew Jerzy Jakubowski, "On the coefficients of starlike functions of some classes," Buzz. Acad. Polon. Sci. Sér. Sci. Math. Astronom. Phys. 19 (1971), 811-815.

[2] Zbigniew Jerzy Jakubowski, "On the coefficients of starlike functions of some classes," Ann. Polon. Math. 26 (1972), 305-313.

[3] Y. J. Kim and E. P. Merkes, "On certain convex sets in the space of locally schlicht functions," Trans. Amer. Math. Soc. 196 (1974), 217-224.

[4] Vinod Kumar and S. L. Shukla, "Jakubowski starlike integral operators," J. Austral. Math. Soc. (Ser. A), 37 (1984), 117-127.

[5] R. K. Pnadey and G. P. Bhargava, "On convex and starlike univalent functions", BulZ. Austral. Math. Soc. 28 (1983), 393-400.

\author{
Dr. Vinod Kumar \\ Department of Mathematics \\ Christ Church College \\ Kanpur-208001, India. \\ Dr. S. L. Shukla \\ Department of Mathematics \\ Janta College \\ Bakewar-206124 \\ Etawah, India.
}

